\title{
Automated induction thermography for in-line inspection of CFRP
}

\author{
Andrew C.Y. Ngo ${ }^{1, *}$, W. Guo ${ }^{1}$ and V. Kumar ${ }^{1}$ \\ *Email : ngocya@imre.a-star.edu.sg \\ ${ }^{1}$ Structural Materials Department, Institute of Materials Research and Engineering (IMRE), Singapore
}

\begin{abstract}
Compared with other active thermography techniques, induction thermography has the advantages of providing instantaneous results yet not affected by surface conditions such as emissivity or geometry. Hence, this technology is promising for applications such as automated in-line inspection. This work will study the feasibility of using automated induction thermography for defect detection on a defective carbon fiber reinforced polymer (CFRP) composite under different moving speed. The final optimized configuration will be presented after considering the signal-to-noise ratio (SNR) and inspection speed.
\end{abstract}

\section{Introduction}

The three most commonly applied active thermography techniques are flash thermography, induction thermography (or sometimes termed as eddy current pulsed thermography), and laser thermography [1].

As the name suggests, flash thermography makes use of flash lamps to apply instantaneous optical energy onto the surface, which converted into heat and travelled vertically into the component. The cooling pattern of the surface temperature is monitored and that reflects the subsurface condition [1]. Because of the working principle, flash thermography is mostly used for subsurface damage inspection. However, surface conditions such as reflectivity and contamination will affect the inspection results.

Laser thermography uses a point laser source to generate a localized point heating, which disperses laterally to the surrounding area. The thermal dispersion pattern will be deformed if there is a surface crack in the vicinity [1]. Hence, laser thermography is mostly used for surface damage inspection with limited resolution as the crack has to be long enough to create an impact that is significant to be captured by an infrared camera. The inspection results will also be affected by surface geometry.

Unlike the previous two techniques, induction thermography uses high frequency alternating current to induce eddy current in the component, which generates heat due to Joule's Law [2]. As such, the thermal signatures are not affected by material reflectivity/emissivity and is indifferent to surface geometry. In addition, sub-surface defects can produce sufficient thermal contrast and thus be detected.

Due to the short excitation duration and small number of thermal patterns required, this technique can be easily configured for automated in-line inspection - which is a critical enabler when it comes to large area inspection [3]. An example will be the composite aircraft inspection.

Last but not least, the heat is generated in the sample within the skin depth according to Eq. (1)

$$
\delta=\frac{1}{\sqrt{\pi \mu \sigma f}}
$$

where $\delta$ is the skin depth, $\mu$ is the material magnetic permeability, $\sigma$ is the electrical conductivity, and $f$ is the excitation frequency. According to Eq. (1), the skin depth for CFRP composite is $25 \mathrm{~mm}$ when using a $300 \mathrm{kHz}$ excitation frequency [4] and $\sim 50 \mathrm{~mm}$ when using a $100 \mathrm{kHz}$ excitation frequency [5]. Therefore, induction thermography is promising for sub-surface defect inspection in CFRP composite and this work will study systematically the detectability on a range of sub-surface defects with different depth and size under different moving speed.

\section{Methodology}

For most industrial applications, only one-side can be accessed. Hence, induction thermography in the reflection mode will be studied in this work. The component used in this study is a CFRP composite with sub-surface defects with different depth and size. The CFP composite will be placed on a conveyor which can run at a maximum speed of $30 \mathrm{~m} / \mathrm{min}$. Eddy current at various time interval will be activated while the thermal responses will be continuously monitored by the infrared camera. The normalized temperature will be calculated and stich together forming the thermogram to reconstruct the surface condition. The SNR under various study conditions will be one of the criteria to determine the optimized configuration. The other consideration would be the conveyor speed. The results will be summarized in table 1 and the optimized configuration will be presented after considering all the factors.

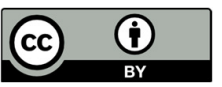


10.21611/qirt.2020.132

$15^{\text {th }}$ Quantitative InfraRed Thermography Conference, 6-10 July 2020, Porto, Portugal

Table 1. SNR from various configurations

\begin{tabular}{|l|l|l|l|l|}
\hline Experiment index & Defect size & Defect depth & SNR & Conveyor speed \\
\hline 1 & & & & \\
\hline 2 & & & & \\
\hline$\ldots$ & & & & \\
\hline
\end{tabular}

\section{Summary}

Compared with other active thermography techniques, induction thermography is not affected by material reflectivity/emissivity, indifferent to surface geometry, provide instantaneous results and is promising for sub-surface defect detection in CFRP. Therefore, induction thermography is a good technique to be automated for in-line inspection of large CFRP components. This work studies the SNR of a CFRP composite sample under different moving speed. The results will provide a guideline to design the system configuration when sub-surface defects of different sizes need to be inspected.

\section{REFERENCES}

[1] Ciampa F., Mahmoodi P., Pinto F., et al., "Recent advances in active infrared thermography for non-destructive testing of aerospace components". Sensors, 18(609), 2018.

[2] He Y., Tian G., Pan M., et al., "An investigation into eddy current pulsed thermography for detection of corrosion blister". Corrosion Science, 78, pp. 1-6, 2014.

[3] Ehlen A., Netzelmann U., Lugin S., et al., "Automated NDT of railway wheels using induction thermography". $55^{\text {th }}$ Annual Conference of the British Institute of Non-Destructive Testing, Nottingham (UK), 2016.

[4] Netzelmann U., Walle G., Ehlen A., et al., "NDT of railway components using induction thermograpy". AIP Conference Proceedings 1706, 150001, 2016.

[5] Netzelmann U., Guo J., "Induction thermography on CFRP and the role of anisotropy", Proceedings of $14^{\text {th }}$ Quantitative InfraRed Thermography conference, Berlin (Germany), 2018. 